\title{
Cirugía de Preservación de Miembros en Tumores Músculo esqueléticos. Utilización de Nitrógeno Líquido. \\ "Una novedosa y económica técnica de reconstrucción" Primera cohorte de pacientes en Uruguay.
}

Limb Preservation Surgery in Musculoskeletal Tumors. Use of Liquid Nitrogen.

"A novel and inexpensive reconstruction technique"

First cohort of patients in Uruguay.

DOI 10.31837/cir.urug/5.1.4

Trabajo que obtuvo el Premio Anual Pedro Larghero -Ernesto Benedek 2020 otorgado por la Sociedad de Cirugía del Uruguay

Gottardo Bianchi ${ }^{1}$

https://orcid.org/0000-0001-8222-4435

Nicolás Casales ${ }^{1}$

https://orcid.org/0000-0003-0318-8654

Claudio Silveri ${ }^{2}$

https://orcid.org/0000-0002-2607-7749

Leticia Gaiero ${ }^{2}$

https://orcid.org/0000-0002-1182-1627

Pablo Stoppiello ${ }^{1}$

https://orcid.org/0000-0003-2085-6968

María Elena Pérez ${ }^{2}$

https://orcid.org/0000-0001-5778-2267

Rogelio Rey ${ }^{1}$

https://orcid.org/0000-0001-5736-3601

Luis Francescoli ${ }^{1}$

https://orcid.org/0000-0002-7048-7630

\footnotetext{
${ }^{1}$ Clínica de Traumatología y Ortopedia Prof. Dr. Luis Francescoli, Facultad de Medicina, Universidad de la República Montevideo, Uruguay. Instituto Nacional de Ortopedia y Traumatología, Administración de Servicios de Salud del Estado, Montevideo, Uruguay bianchigot@hotmail.com

${ }^{2}$ Clínica de Traumatología y Ortopedia Pediátrica, Prof. Dra. María Elena Pérez, Facultad de Medicina, Universidad de la República Montevideo, Uruguay. Centro Hospitalario Pereira Rosell. Administración de Servicios de Salud del Estado, Montevideo, Uruguay
} 


\section{¿Qué es UPOME y por qué?}

Desde el año 2013 en el seno de la clínica de Ortopedia y traumatología de adultos y pediátrica de la UdelaR, con la colaboración de la cátedra de anatomía patológica, imagenología, fisiatría, INCA (Instituto Nacional del Cáncer), Fundación Pérez Scremini comenzamos la unidad de patología oncológica músculo-esquelética (UPOME). Esta unidad tiene el fin de atender a pacientes del sistema público con afecciones oncológicas músculo esqueléticas: los sarcomas óseos y de partes blandas, así como la enfermedad metastásica ósea. Este grupo trata de manera multidisciplinaria la ortopedia oncológica, subdisciplina dentro de la ortopedia. Este trabajo es el fruto de casi 7 años de trabajo.

\section{Resumen}

Introducción: La cirugía conservadora de miembros constituye el tratamiento estándar en el manejo de tumores músculo-esqueléticos. El problema al que se enfrenta el cirujano es la reconstrucción luego de una resección amplia. Existen diversas técnicas como endoprótesis, aloinjertos y autoinjertos, entre otras.

En 1999 en Japón, Tsuchiya, et al desarrollaron la técnica de reconstrucción biológica utilizando nitrógeno líquido (NL). El objetivo del siguiente trabajo consiste en presentar la primera cohorte de pacientes tratados con dicha técnica en nuestro país. Además analizar los siguientes parámetros: recurrencia local, plazos de consolidación, sobrevida del autoinjerto y evaluación funcional al final del seguimiento.

Metodología: Se realizó un estudio retrospectivo desde agosto 2015 hasta junio de 2019 de los pacientes tratados con autoinjerto congelado en NL. Todos fueron evaluados en equipo multidisciplinario y tratados por el mismo equipo quirúrgico. Se registraron un total de 19 casos, se incluyeron 13 para su evaluación, siendo excluidos el resto por tener menos de 6 meses de seguimiento. La técnica consiste en la resección tumoral con márgenes amplios, exposición de la pieza tumoral a un ciclo de inmersión en NL a -196 grados Celcius por 20 minutos, luego calentamiento progresivo por 15 minutos a temperatura ambiente y 10 minutos en agua destilada. Logrando erradicar las células tumorales por crioterapia. Seguido de la reimplantación del hueso congelado y fijación con material de osteosíntesis.

En la evaluación, la recurrencia local fue evaluada clínica e imagenológicamente. La consolidación se evaluó según lo definido por Zekry et al: paciente sin dolor al deambular y líneas de osteotomías no son visibles o existe un callo evidente en 3 de las corticales en los enfoques antero posterior y lateral. La sobrevida del autoinjerto fue evaluada según si hubo necesidad o no de retirarlo.

Resultados: Al final del seguimiento todos los pacientes se encuentran vivos, ninguno de los pacientes con enfermedad localizada ha desarrollado metástasis a distancia. Se registró un 
paciente con recurrencia local en partes blandas. Se logró la consolidación en un $83 \%$ de los pacientes, con una media de 6.7 meses. Fue necesario retirar el injerto en 3 casos, dos debido a una infección profunda y otro por colapso articular. La evaluación funcional según el score MSTS-ISOLS promedio fue de 73,8\% (rango 13-100\%). Dicho promedio fue de 89,9\% (63$100 \%$ ) en los 10 pacientes que se mantuvo el autoinjerto.

Conclusiones: La técnica de autoinjerto congelado en NL constituye una muy buena opción de reconstrucción en tumores músculo-esqueléticos. Es una técnica de fácil acceso, bajo costo y mantiene las propiedades de osteoconducción y osteoinducción. Nuestra serie presenta resultados similares a los estudios publicados en cuanto a recurrencia local, consolidación y score funcional.

\section{Introducción}

Antes de los años 1970 la amputación era el tratamiento de elección en tumores óseos (TO). El aumento de la sobrevida en estos pacientes, debido fundamentalmente al uso de la quimioterapia (QT), así como el avance y desarrollo en los métodos de imagen, el trabajo en equipo multidisciplinario especializados, y el desarrollo de nuevos procedimientos quirúrgicos, han permitido que en la actualidad el tratamiento quirúrgico estándar sea la cirugía conservadora de miembros ${ }^{1,2}$.

Luego de una resección esquelética amplia, el cirujano se enfrenta al problema de la reconstrucción de grandes defectos óseos creados por la resección tumoral. Existen distintas opciones reconstructivas ${ }^{3}$.

Existen métodos no biológicos: las endoprótesis tumorales ${ }^{4}$, y métodos biológicos: como los aloinjertos $^{5,6}$, aloprotesis ${ }^{7}$, peroné vascularizado $^{8}$, distracción osteogénesis $^{9}$, o la reutilización del segmento resecado y esterilizado (autoinjerto) con distintas técnicas como: pasteurización ${ }^{10}$, irradiación ${ }^{11}$, autoclave ${ }^{12}$ y recientemente el uso de nitrógeno líquido (NL) ${ }^{13}$.

El primer autor en utilizar el NL en cirugía fue Cooper en 1962, su uso fue en cirugía a nivel de los ganglios basales ${ }^{14}$. Desde entonces la criocirugía se ha utilizado en cirugía oncológica en distintas especialidades.

En oncología ortopédica, en el año 1964 en el Hospital Memorial Sloan-Kettering de Estados Unidos se registra el primer uso del $\mathrm{NL}^{15}$. Luego diversos autores han utilizado el NL como adyuvante en el manejo de TO. No obstante, ninguno de los autores utilizó el NL como método de esterilización tras la resección en bloque del tumor y su posterior reimplantación.

Por motivos socio-culturales y religiosos en países asiáticos la mayoría de la población no acepta el aloinjerto como método de reconstrucción, sumado al difícil acceso a las prótesis tumorales, se ha motivado el desarrollo de nuevas alternativas terapéuticas. Es así que en el año 1999 el profesor H.Tsuchiya junto a su equipo en la Universidad de Kanazawa, Japón, desarrolló la técnica de autoinjerto esterilizado mediante congelamiento en NL como método de reconstrucción en defectos óseos en $\mathrm{TO}^{13}$. 
El desarrollo de este método se basó en estudios in vitro e in vivo de las propiedades del NL sobre la proliferación celular del osteosarcoma ${ }^{16}$.

\section{Objetivos}

\section{- Generales:}

El objetivo del presente trabajo es exponer esta novedosa técnica, destacando la aplicación de la misma en nuestro país. Además se presentará la primera cohorte en el Uruguay de pacientes con tumores músculo esqueléticos tratados mediante la reconstrucción con autoinjerto congelado en NL.

\section{- Específicos:}

Analizar recurrencia local (RL), plazo de consolidación y durabilidad en el tiempo de la reconstrucción.

Evaluación funcional de los pacientes al final del seguimiento, según el score de la MSTS/ISOLS ${ }^{17}$.

\section{Metodología}

Presentamos un trabajo retrospectivo desde agosto del 2015 hasta junio del 2019. Todos los pacientes incluidos fueron evaluados, discutidos y tratados en equipo multidisciplinario por la unidad de patología oncológica músculo-esquelética (UPOME). Esta incluye: anatomía patológica, imagenología, oncología, radioterapia, cirugía plástica, psicología y ortopedista oncológico. En dicho período de tiempo, un total de 19 pacientes fueron sometidos a la técnica quirúrgica antes mencionada.

Indicaciones: esta técnica es utilizada para la reconstrucción luego de la resección tumoral en cirugía oncológica músculo- esquelética. Se pueden tratar pacientes tanto niños como adultos con TO primarios o metastásis de los miembros pelvis y sacro y columna. La mejor indicación corresponde a tumores osteoblásticos (formadores de hueso) aunque en tumores con lesiones líticas se puede añadir cemento como método adyuvante para el relleno de la cavidad.

Incluimos en este trabajo todos los pacientes sin límite de edad: niños y adultos. Se excluyeron los pacientes con menos de 6 meses de seguimiento. Quedando de los 19 pacientes, 13 casos incluidos para la evaluación.

La evaluación inicial fue realizada según el protocolo de nuestra institución para pacientes con tumores músculo-esqueléticos. La misma consiste en evaluación y registro clínico inicial, estudios de laboratorio (hemograma, fosfatasa alcalina, LDH) e imagenológico (en lo local: Rx, TC y RNM del segmento afectado y en lo sistémico: centellograma óseo, TC de tórax abdomen y pelvis). Luego fue planificada y efectuada una biopsia con aguja gruesa en todos los casos. Una 
vez confirmado el diagnóstico histológico se procedió al tratamiento sistémico (en el caso que corresponda) seguido de la cirugía. Todos fueron operados por el mismo equipo quirúrgico. En todos los casos fueron informados acerca del procedimiento, ventajas y desventajas así como otras opciones terapéuticas, y se realizó consentimiento informado.

En el postoperatorio, la evaluación radiográfica fue realizada al mes, 3 meses, 6 meses, 1 año y luego anual. El seguimiento clínico se realizó caso a caso según la evolución en el postoperatorio. En cuanto al seguimiento sistémico, la evaluación se realizó dependiendo del tipo histológico tumoral.

Los controles postoperatorios fueron dirigidos al estudio de la consolidación del injerto, detección de posibles recidivas, diseminación metastásica y evaluación funcional. La consolidación se estudio con radiografías simples. La búsqueda de recidivas fue evaluada mediante Rx y RNM. La diseminación a distancia se evaluó mediante tomografía de tórax abdomen y pelvis. Estos estudios fueron analizados por los dos cirujanos ortopedistas oncológicos y el equipo de imagenología especializado en músculo esquelético.

Para la consolidación usamos la definición establecida por Zekry et a ${ }^{18}$ quien define como unión cuando el paciente no tiene dolor al deambular y cuando las líneas de osteotomías no son visibles o existe un callo evidente en 3 de las corticales en los enfoques antero posterior y lateral.

La técnica quirúrgica consiste en realizar una resección amplia del tumor con márgenes oncológicos, una vez obtenida la pieza tumoral, se remueven los tejidos blandos los cuales son enviados a anatomía patológica para recuento de necrosis si corresponde. Se deben realizar perforaciones en el tejido óseo que será inmerso en el NL para evitar que sufra una fractura al ser congelado, éste es un paso crítico a tener en cuenta para evitar dañar la pieza que más tarde será utilizada como injerto.

Además, dichas muestras sirven como material a ser enviado para el estudio anatomo-patológico, para evaluar la respuesta a la quimioterapia, el índice de necrosis que es un factor pronóstico.

Luego se procede a la esterilización de la pieza mediante un ciclo de inmersión en NL a -196 grados Celsius por 20 minutos, realizando una inmersión cuidadosa y progresiva, a continuación, la pieza congelada se expone por 15 minutos a temperatura ambiente para lograr un calentamiento progresivo. Por último, se coloca por 10 minutos en agua destilada.

Una vez culminado este procedimiento la pieza así esterilizada es reimplantada y fijada en el defecto óseo creado, mediante alguna técnica de osteosíntesis y/o la asociación con una prótesis ("compuesto"). 


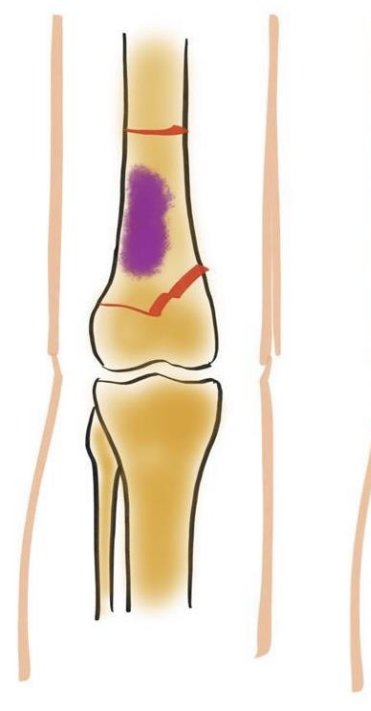

Resección

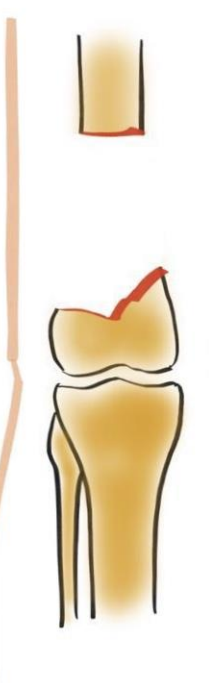

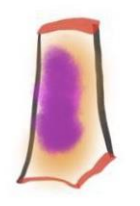
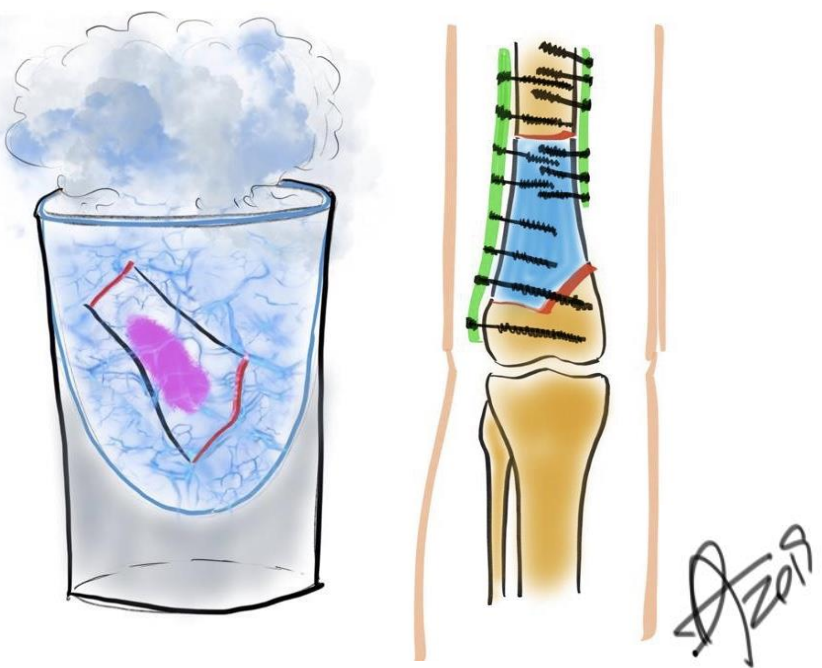

Re-Implantación

Figura 1. Esquema de la técnica quirúrgica de esterilización en NL. Tipo de congelamiento libre. Tipo de resección Intercalar. Se realiza la resección amplia del tumor, la pieza se retira y se sumerge durante 20 minutos a -196 grados Celsius en NL para lograr erradicar las células tumorales. Luego del calentamiento progresivo dicha pieza es reimplantada (conformando un autoinjerto) y se fija con osteosíntesis (placas y tornillos en este esquema).

Los tipos de resección y por consiguiente el tipo de injerto a utilizar puede ser tanto intercalar (segmento óseo sin comprometer la superficie articular), hemiresección (resección de una sola cortical) o de tipo osteoarticular (OA): el cual incluye la superficie articular. En este último caso es posible la re sutura de estructuras capsulo-ligamentarias.

Son posibles dos tipos de congelamientos. El primero denominado "libre" (Fig. 1) en el cual se extrae toda la pieza para ser esterilizada en NL y luego es reimplantada. El segundo tipo 
denominado "pediculado" (Fig. 2) requiere sólo una osteotomía o luxación del sitio articular involucrado, quedando el segmento a tratar vinculado al resto del miembro.
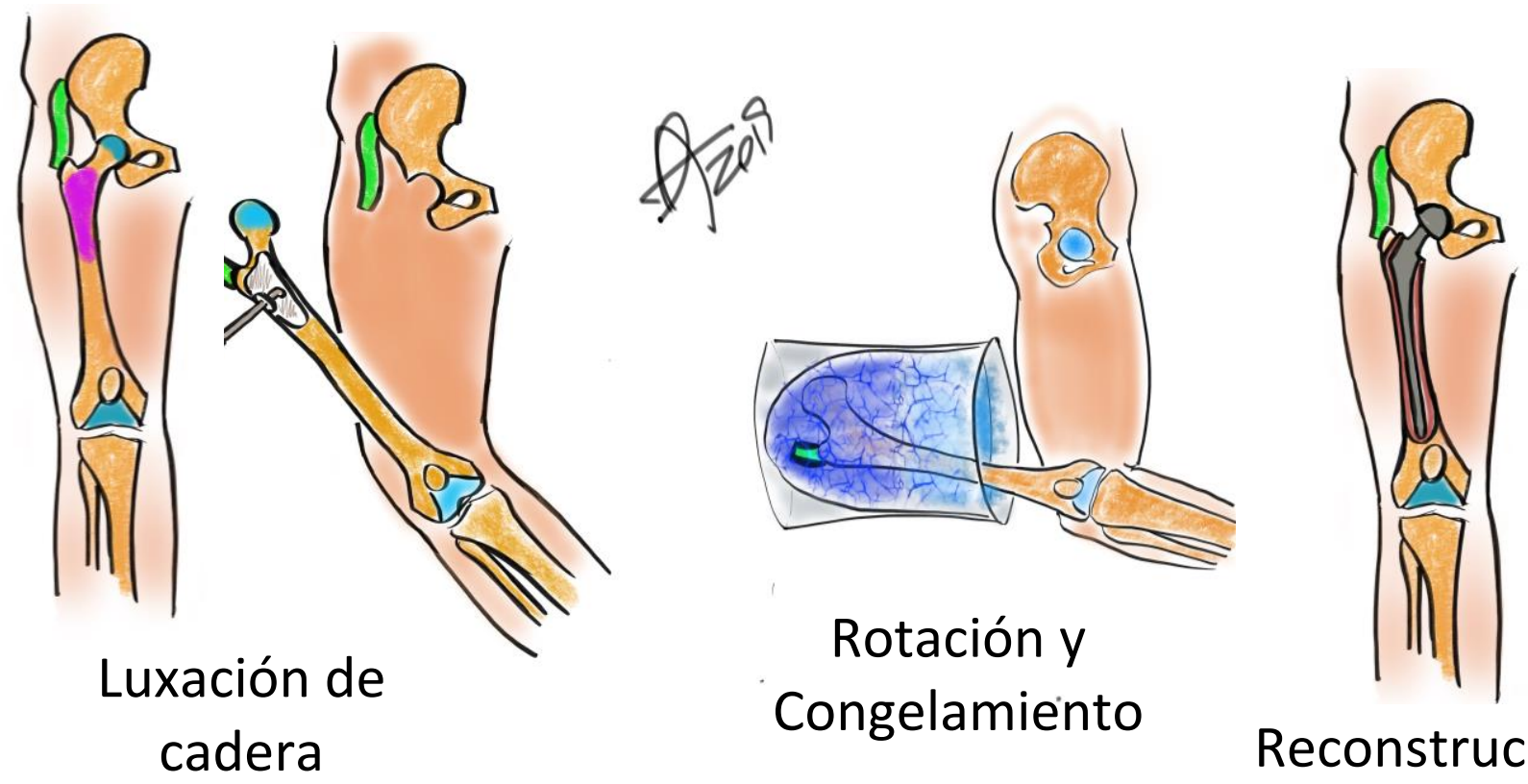

Reconstrucción

Figura 2. Esquema del método de congelamiento "pediculado". En este ejemplo una lesión tumoral del fémur proximal. Luego del abordaje se realiza una luxación de la cadera respetando los márgenes del tumor. Se realiza una rotación del miembro con lo cual se introduce el fémur proximal y el sector óseo que involucra el tumor en un recipiente con NL durante 20 minutos, luego del calentamiento progresivo a temperatura ambiente y en agua destilada, se rota nuevamente la cadera, se coloca una prótesis de fémur proximal y se deja la cadera reducida en el cotilo.

Se presentan tablas y gráficos de frecuencia para la descripción de variables cualitativas, así como medidas de resumen para las continuas. En el estudio de asociación se usó test Chi cuadrado o exacto de Fisher en los casos en que alguna de las celdas esperadas presente valores menores a 5. El nivel de significación se fijó en 0,05. El software utilizado correspondió a STATA v. 12.0

\section{Resultados}

Se analizan 13 pacientes que cumplen con criterios de inclusión y exclusión. El detalle del registro de los pacientes se encuentra en la tabla 1.

El estudio incluyó 13 pacientes, 10 hombres y 3 mujeres. El promedio de edad correspondió a 28.1 (SD 4.7) con un mínimo y un máximo en 5 y 57 años respectivamente. El 76,9\% (10) de los pacientes son de sexo masculino, existiendo una razón de 3,3 hombres/mujer. En el caso del sexo 
femenino el promedio de edad fue de 41,0 +/- 7,6 años, mientras que en los hombres fue de 24,2 $+/-5,2$ años.

En cuanto a la localización: el hueso más afectado fue el fémur. El tipo de tumor mas frecuente fue el osteosarcoma con 6 casos. El seguimiento medio es de 27 meses (rango 7-46). Ningún paciente falleció y ningún paciente se ha perdido en el seguimiento.

El 46,2\% (6) de los pacientes recibieron QT. Estos corresponden a 2 sarcomas de Ewing, 1 osteosarcoma perióstico, 1 osteosarcoma Telangiectásico, 1 osteosarcoma Convencional y 1 "skip" metástasis de osteosarcoma. El plan de QT para osteosarcoma difiere del utilizado en el sarcoma de Ewing.

En todos los casos de osteosíntesis rígida el método de fijación consistió en placa bloqueada y tornillos. Se utilizó en dos casos cemento para el relleno de la lesión (caso 6 y 8), debido a que se trataba de lesiones líticas.

En cuanto al método de congelamiento el más frecuente fue el libre en el 92,3\% (12) de los pacientes. El tipo de injerto más frecuente fue el intercalar con 6 casos.

La longitud promedio fue de $15.2 \mathrm{~cm}$ (SD 1.2, rango 5.0-28.0). En el caso del fémur la longitud fue de 18,0 +/- 2,2 cm. Para la tibia fue de 12,4 +/- 2,6 cm. Para los estadíos M0 la longitud promedio fue de 16,1 +/- 1,6 cm, para los M1 fue de 10,0 +/- 5,0 cm. Para los osteosarcomas fue de $17,2+/-2,4 \mathrm{~cm}$.

Del total, sólo dos casos (11 y 12) correspondieron a pacientes metastásicos (M1), de los no metastásicos (M0) ninguno ha desarrollado enfermedad metastásica hasta el último seguimiento.

En 3 casos (3, 6 y 10) se tuvo que remover el autoinjerto.

En cuanto a las complicaciones, fueron registradas en 8 pacientes. De éstas, 3 de ellas podemos catalogarlas como complicaciones menores. El caso 1 presentó un trazo de fractura sin desplazamiento debajo del injerto dado que realizó carga de peso antes de lo indicado, fue tratado con yeso y consolidó sin complicaciones. El caso 5 presentó una infección superficial que mejoró con tratamiento antibiótico intravenoso y cámara hiperbárica. El paciente número 8 presentó una discrepancia de longitud de MMII la cual fue tratada satisfactoriamente con epifisiodesis del miembro contralateral, dicha complicación fue esperada que sucediera al año y seis meses de postoperatorio, momento en el cual fue realizada la epifisiodesis.

En cuanto a las complicaciones mayores, el paciente número 3 sufrió un colapso del injerto OA y una infección profunda, lo que motivó el retiro del autoinjerto 2 años y 9 meses luego de la cirugía inicial.

El caso 6 evolucionó con un colapso del injerto y desaxación en varo con dismetría del miembro afectado. Fue retirado el autoinjerto 2 años y 2 meses de la cirugía inicial.

La paciente del caso 10 presentó en el postoperatorio una necrosis de piel en la región distal de la pierna con exposición del autoinjerto, resultando en una infección profunda, requiriendo el retiro del mismo 4 meses postoperatorio.

Por su parte el paciente número 9 presentó un seroma postoperatorio inmediato siendo tratado con drenaje quirúrgico. Tres semanas más tarde evolucionó con una dehiscencia de la herida 
Tabla 1. Detalle de los datos de los pacientes incluidos en este trabajo.

QT: Quimioterapia. M: masculino. F: femenino. Prox: proximal. Prof: Profunda. OA: Osteoarticular. Score: Score de MSTS-ISOLS ${ }^{15}$

\begin{tabular}{|c|c|c|c|c|c|c|c|c|c|c|c|c|}
\hline Paciente & $\begin{array}{l}\text { Edad/ } \\
\text { Sexo }\end{array}$ & Sitio & Estadio & Histología & $\begin{array}{c}\text { Tipo } \\
\text { Injerto }\end{array}$ & $\begin{array}{c}\text { Método } \\
\text { Congelamien } \\
\text { to }\end{array}$ & $\begin{array}{l}\text { Longitud } \\
\quad(\mathbf{c m})\end{array}$ & QT & $\begin{array}{c}\text { Unión } \\
(\mathbf{m})\end{array}$ & $\begin{array}{c}\text { Score } \\
(\%)\end{array}$ & $\begin{array}{c}\text { Seguimie } \\
\text { nto } \\
(\mathrm{m})\end{array}$ & Complicaciones \\
\hline 1 & 16. M & $\begin{array}{l}\text { Tibia } \\
\text { Diáfisis }\end{array}$ & M0 & $\begin{array}{l}\text { Osteosarcoma } \\
\text { Periostico }\end{array}$ & Hemicortical & Libre & 12 & $\mathrm{Si}$ & 6 & 100 & 46 & $\begin{array}{l}\text { Fractura debajo del } \\
\text { injerto }\end{array}$ \\
\hline 2 & 29. F & $\begin{array}{l}\text { Fémur } \\
\text { Diáfisis }\end{array}$ & M0 & $\begin{array}{l}\text { Osteosarcoma } \\
\text { Parostal }\end{array}$ & Intercalar & Libre & 19 & No & 9 & 97 & 40 & No \\
\hline 3 & 13. $\mathrm{M}$ & $\begin{array}{l}\text { Fémur } \\
\text { Prox. }\end{array}$ & M0 & Ewing & $\mathrm{OA}$ & Libre & 18 & $\mathrm{Si}$ & 8 & 17 & 40 & $\begin{array}{l}\text {-Colapso Injerto } \\
\text {-Infección Prof. }\end{array}$ \\
\hline 4 & 25. M & $\begin{array}{l}\text { Fémur } \\
\text { Distal }\end{array}$ & M0 & $\begin{array}{l}\text { Osteosarcoma } \\
\text { Parostal }\end{array}$ & Intercalar & Libre & 15 & No & No & 83 & 39 & $\begin{array}{l}\text {-Recidiva en PB } \\
\text {-Seudoartrosis }\end{array}$ \\
\hline 5 & 57. M & $\begin{array}{l}\text { Tibia } \\
\text { Diáfisis }\end{array}$ & M0 & Adamantinoma & Intercalar & Libre & 20 & No & 7 & 100 & 37 & Infección Superf. \\
\hline 6 & 5. M & $\begin{array}{l}\text { Fémur } \\
\text { Distal }\end{array}$ & M0 & $\begin{array}{c}\text { Osteosarcoma } \\
\text { Telangiectasico }\end{array}$ & OA & Libre & 13 & $\mathrm{Si}$ & 6 & 30 & 34 & $\begin{array}{l}\text {-Desaxación } \\
\text {-Colapso Injerto }\end{array}$ \\
\hline 7 & 55. F & $\begin{array}{l}\text { Humero } \\
\text { Prox. }\end{array}$ & M0 & Condrosarcoma & Composite & Libre & 12 & No & 6 & 100 & 31 & No \\
\hline 8 & 10. M & $\begin{array}{l}\text { Tibia } \\
\text { Distal }\end{array}$ & M0 & $\begin{array}{l}\text { Osteosarcoma } \\
\text { Convencional }\end{array}$ & $\mathrm{OA}+$ Artodesis & Libre & 16 & $\mathrm{Si}$ & 7 & 83 & 26 & Discrepancia MMII \\
\hline 9 & 13. $\mathrm{M}$ & Pelvis & M0 & Ewing & Intercalar & Libre & 15 & $\mathrm{Si}$ & 7 & 63 & 18 & $\begin{array}{c}\text {-Seroma } \\
\text {-Dehiscencia herida }\end{array}$ \\
\hline 10 & 39. $\mathrm{F}$ & $\begin{array}{l}\text { Tibia } \\
\text { Distal }\end{array}$ & M0 & Condrosarcoma & Intercalar & Libre & 9 & No & No & 13 & 14 & $\begin{array}{l}\text {-Necrosis de piel } \\
\text {-Infección Prof. }\end{array}$ \\
\hline 11 & 28. M & $\begin{array}{l}\text { Fémur } \\
\text { Prox. }\end{array}$ & M1 & $\begin{array}{c}\text { Metástasis } \\
\text { Sarcoma Epiteloide }\end{array}$ & Composite & Pediculado & 15 & No & - & 97 & 11 & No \\
\hline 12 & 43. M & $\begin{array}{l}\text { Tibia } \\
\text { Prox }\end{array}$ & M1 & $\begin{array}{l}\text { Skip Metastasis } \\
\text { Osteosarcoma }\end{array}$ & Hemicortical & Libre & 5 & $\mathrm{Si}$ & 4 & 93 & 8 & No \\
\hline 13 & 32. M & $\begin{array}{l}\text { Fémur } \\
\text { Diáfisis }\end{array}$ & M0 & $\begin{array}{c}\text { Osteosarcoma } \\
\text { Central de Bajo } \\
\text { Grado }\end{array}$ & Intercalar & Libre & 28 & No & 7 & 83 & 7 & No \\
\hline
\end{tabular}

quirúrgica y exposición parcial del autoinjerto correspondiente a la cresta ilíaca, fue tratado con resección parcial del injerto y cierre de la herida, evolucionando favorablemente.

El caso 4 no logró la consolidación de la osteotomía proximal a pesar de 2 cirugías de aporte de injerto de cresta ilíaca. Además, este paciente 2 semanas antes de la finalización del estudio (3 años y 3 meses posteriores a la primera cirugía) desarrolló una recidiva local en partes blandas.

Si estudiamos la relación entre haber recibido PQT y la presencia de complicaciones postquirúrgica, se observa que no se encontró una asociación estadísticamente significativa entre ambas, valor $\mathrm{p}=0,179$.

\begin{tabular}{|ll|l|l|l|}
\hline & & \multicolumn{2}{|l|}{ Complicaciones } & \multirow{2}{*}{ Total } \\
\cline { 3 - 5 } & & No & Si & \\
\hline PQT & No & 4 & 3 & 7 \\
& Si & 1 & 5 & 6 \\
Total & & 5 & 8 & 13 \\
\hline
\end{tabular}


No se evidenció ninguna recidiva en el autoinjerto.

En relación con los meses necesarios para lograr la unión, se encontró que en 2 pacientes no se logró, en los restantes el promedio para conseguirlo fue de 6,7 +/- 1,0 meses con un mínimo y máximo en 4 y 9 meses respectivamente. Cabe aclarar que el paciente número 11 no se incluye en la evaluación de la consolidación dado que al tratarse de un "composite" pediculado no requiere la consolidación entre autoinjerto y hueso sano.

La evaluación funcional según el score MSTS-ISOLS ${ }^{17}$ promedio fue de 73,8\% (rango 13$100 \%$ ). Dicho promedio fue de $89,9 \%$ (63-100\%) en los 10 pacientes que se mantuvo el autoinjerto.

\section{Discusión}

Existen diversos métodos para la reconstrucción del defecto óseo creado luego de una resección amplia en patología tumoral. Todos se presentan con ventajas y desventajas. Las endoprótesis muestran buenos resultados funcionales sobre todo a corto y mediano plazo ${ }^{19}$. Entre sus desventajas se destaca su alto costo, escasa disponibilidad en algunos centros, y alta tasa de revisión, sobre todo en pacientes pediátricos, grupo etario donde se registran la mayoría de los TO primarios ${ }^{19,20}$.

Los aloinjertos presentan la desventaja de la necesidad de un banco de hueso con material a medida, además de la potencial transmisión de enfermedades, en algunos casos la no aceptación por parte de los pacientes ${ }^{21,22}$. En nuestro país actualmente no contamos con un banco de hueso que tenga segmentos óseos a medida para todos los pacientes.

La técnica de osteogenesis por distracción presenta la desventaja de ser un tratamiento prolongado, complejo y requiere múltiples procedimientos quirúrgicos ${ }^{9}$.

El autoinjerto congelado en NL, se presenta como una solución biológica cuyas ventajas incluyen: bajo costo, fácil acceso (es el propio hueso del paciente: autoinjerto), logra una erradicación completa del tumor, preserva la actividad de las proteínas morfogenéticas óseas (ésta actividad estaría más conservada en la técnica de congelamiento respecto a pasteurización y autoclavado ${ }^{23}$ ), mantiene la capacidad de osteoconducción y osteoinducción, presenta un encastre perfecto entre el injerto y el hueso sano, no requiere un stock de banco de hueso, permite la re fijación de tendones y ligamentos, no tiene el posible riesgo de transmisión de enfermedades ni tiene problemas de rechazo del injerto.

Técnicamente es un método versátil, los autores que describieron la técnica original, fueron a lo largo del tiempo desarrollando mejoras en la misma para extender su uso. Por ejemplo, es posible la combinación del autoinjerto congelado más prótesis ("compuesto" ${ }^{24}$ ), esta opción surge en respuesta a los cambios degenerativos y colapso en la superficie articular observados al uso del injerto OA. En nuestra casuística tenemos dos casos en los cuales realizamos el tipo de injerto compuesto.

Otra mejora en la técnica que los autores describen es una variante en el tipo de congelamiento denominado pediculado. El mismo surge con el objetivo de mejorar los plazos en la 
consolidación del autoinjerto libre. Esta técnica lograría una recuperación funcional precoz con un menor índice de complicaciones ${ }^{25-26}$.

Entre sus desventajas se destaca la imposibilidad de acceder al total de la pieza para su análisis histológico y valoración de la respuesta a la QT, no obstante, se considera que la muestra que se obtiene en la resección de la masa extraesquelética y óseas previas al congelamiento son representativas ${ }^{27}$.

Esta técnica logra una necrosis total de las células tumorales ${ }^{16}$, además no muestra diferencias significativas en cuanto a la resistencia a la compresión respecto al hueso normal, lo que asegura una suficiente resistencia inicial para la reconstrucción, comparable o incluso superior al aloinjerto $^{16}$. Hay que destacar que dicha resistencia del autoinjerto se refiere cuando la lesión es blástica, disminuyendo su resistencia en lesiones líticas en las cuales habría q evitar el uso del autoinjerto o colocar algún adyuvante como el cemento óseo. Que el injerto congelado logre mantener propiedades mecánicas es un aspecto de suma importancia ya que el mismo será utilizado para resistir carga de peso cuando es utilizado en miembros inferiores.

La exposición del hueso tumoral al NL es capaz de desarrollar inmunidad celular y humoral (crioinmunidad), siendo capaz de reducir tamaño y número de metástasis. Esta es una de las propiedades y ventajas más prometedoras de esta técnica teniendo un efecto sistémico ${ }^{28,29,30}$.

Tabla 2. Resultados de los casos descritos en la literatura. Comparación con nuestra casuística. OA: osteoarticular; m: meses.

\begin{tabular}{|c|c|c|c|c|c|}
\hline Estudio & $\begin{array}{c}\text { Tsuchiya et al. } \\
\qquad(2005)\end{array}$ & $\begin{array}{c}\text { Rahman et } \\
\text { al. } \\
(2009)\end{array}$ & $\begin{array}{c}\text { Igarashi et } \\
\text { al. } \\
(2014)\end{array}$ & $\begin{array}{c}\text { Paholpak et } \\
\text { al. } \\
(2015)\end{array}$ & $\begin{array}{c}\text { Nuestro } \\
\text { estudio } \\
(2019)\end{array}$ \\
\hline Pacientes & 28 & 10 & 36 & 12 & 13 \\
\hline Seguimiento medio (m) & $28.1(10-54)$ & 54 & $101(16-163)$ & $32(12-60)$ & $27(7-46)$ \\
\hline Unión (\%) & 93 & 100 & 72 & 100 & 83 \\
\hline Unión media (m) & 6.7 & 8.8 & 6.2 & 8.2 & 6.7 \\
\hline No unión (\%) & 7.1 (2 ptes.) & 0 & 16.6 (6 ptes.) & 0 & 16.7 (2 ptes.) \\
\hline Recurrencia Local & 2 & 0 & 4 & 1 & 1 \\
\hline $\begin{array}{l}\text { Sobrevida del Injerto } \\
(\%)\end{array}$ & 96.4 & 100 & 80.6 & 83.3 & 77 \\
\hline Score MSTS-ISOLS & 71.4 & 82.4 & 72.2 & 79 & 73.8 \\
\hline Tipo de Injerto & $\begin{array}{l}14 \text { OA } \\
9 \text { Intercalar } \\
2 \text { Articulación } \\
\text { completa }\end{array}$ & $\begin{array}{l}6 \text { Intercalar } \\
4 \mathrm{OA}+ \\
\text { artrodesis }\end{array}$ & $\begin{array}{l}16 \text { OA } \\
13 \text { Intercalar } \\
7 \text { Compuesto }\end{array}$ & $\begin{array}{l}8 \mathrm{OA} \\
4 \text { Intercalar }\end{array}$ & $\begin{array}{l}6 \text { Intercalar } \\
2 \text { OA } \\
2 \text { Hemicortical } \\
2 \text { Composite } \\
1 \text { OA + artrodesis }\end{array}$ \\
\hline
\end{tabular}


Tanzawa et $\mathrm{al}^{31}$ informan sobre el examen histológico de una pieza tratada con NL removida 6 años luego de la esterilización, no encontrando células tumorales, y destacan la presencia de osteocitos y microvasos en la corteza y medula ósea, así como áreas focales de condrocitos en la superficie articular, evidenciando una revitalización del injerto ${ }^{31}$.

Los autores elaboramos una tabla con datos de interés de los pacientes, comparado con datos de la literaratura (Tabla 2).

Del análisis de los estudios reportados en la bibliografía, valoramos recurrencia local, consolidación, sobrevida del autoinjerto.

En cuanto a la recurrencia local, Paholpak et $\mathrm{al}^{32}$ describe 1 paciente $(8.3 \%)$, apareciendo a los 28 meses de seguimiento. Tsuchiya et $\mathrm{al}^{13}$ informa dos pacientes $(7.1 \%)$ con RL y aclara que dicha recurrencia se produjo en partes blandas, siendo tratados satisfactoriamente con una nueva resección amplia. Igarashi et $\mathrm{al}^{33}$ tuvo 4 pacientes $(11.1 \%)$ con $\mathrm{RL}$. El autor señala que ésta se dio en tejidos blandos distantes del autoinjerto y próximo a estructuras neurovasculares, no siendo observada la invasión del autoinjerto. Se realizó en todos los casos resección amplia de la recidiva sin tener que realizar procedimientos sobre el autoinjerto.

En nuestra casuística encontramos un caso $(7.7 \%)$ con RL en tejidos blandos, 39 meses postoperatorio. Este resultado es similar a lo reportado en la bibliografía (Tabla 2). Los autores antes mencionados, proponen que las recidivas están vinculadas a una inadecuada resección de las partes blandas y no vinculadas a la técnica de congelamiento.

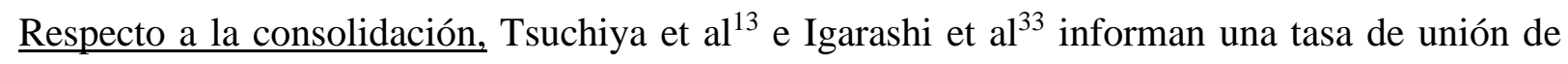
$93 \%$ y $72 \%$ en un plazo de 6.7 y 6.2 meses respectivamente. Por su parte Rahman et $\mathrm{al}^{34}$ y Paholpak et $\mathrm{al}^{32}$ informan un $100 \%$ de consolidación en plazos de 8.8 y 8.2 meses respectivamente.

De los 2 pacientes que no lograron la unión según Tsuchiya et $\mathrm{al}^{13}$, un paciente falleció por la enfermedad, antes de lograr la consolidación, tratándose de un paciente de 17 años con osteosarcoma metastásico (M1) de fémur distal. La otra paciente fue retirado el injerto antes de la unión debido a una infección postoperatoria. Igarashi et $\mathrm{al}^{33}$ reporta 6 pacientes que no lograron la consolidación inicialmente, siendo tratados con injerto de cresta ilíaca con o sin fijación con placa adicional, dichos procedimientos se llevaron a cabo en una media de 18.3 meses ( 7 a 33) luego de la cirugía inicial, logrando la consolidación en todos con una media de 5.5 meses ( 2 a 8) luego de la segunda cirugía.

En nuestra casuística, se logró la consolidación en un 83\%, con una media de 6.7 meses. Estos datos son muy similares a los reportados por los estudios previos (tabla 2). De los dos pacientes que no lograron la consolidación, un caso fue debido a que se tuvo que retirar el injerto precozmente (4 meses) dado una infección profunda, el otro caso fue tratado mediante la colocación de autoinjerto de cresta iliaca no habiendo lograda la consolidación hasta el último seguimiento.

Sobrevida del Autoinjerto:

Igarashi et al $^{33}$ obtuvo una sobrevida del autoinjerto en 29 de 36 pacientes $(80.6 \%)$, siendo de $86.1 \%$ a los 5 años y $80.6 \%$ a 10 años. Definió como sobrevida del injerto, aquel que se logró 
conservar y fue funcional, por su contraparte lo catologó como "muerto" cuando fue removido o sufrió re-absorción no siendo funcional. El autor destaca que los 7 injertos removidos correspondieron a injertos osteoarticulares (OA) y todos fueron tratados exitosamente mediante conversión a endoprótesis. Dado lo antes mencionado el autor recomienda fuertemente el uso de "composite" (autoinjerto más prótesis) en aquellos tumores con gran extensión en la superficie articular. Paholpak et al $^{32}$ informa dos pacientes del total en los cuales tuvo que retirar el autoinjerto, el primero a los 51 meses por una fractura del autoinjerto, requiriendo una endoprotesis. El segundo a los 20 meses debido a una lisis.

En ambos pacientes el tipo de autoinjerto fue OA. Tsuchiya et al $^{13}$ del total de 28 casos informa sólo un paciente en el cual tuvo que retirar el injerto, debido a infección. También se trató de un autoinjerto OA.

Nuestra serie mostró una sobrevida del autoinjerto del $77 \%$ al final del seguimiento. Fue necesario retirar el autoinjerto en 3 pacientes. De éstos, el paciente número 3 fue retirado debido a una infección profunda a los 33 meses de postoperatorio. Fue tratado con un espaciador de cemento en fémur proximal y actualmente está en tratamiento con la técnica de fijadores externos circulares mediante soporte pélvico asociado a osteotomía distal de alargamiento.

El caso número 6 se trata de un paciente de 5 años portador de un osteosarcoma telangiectásico del femur distal derecho, fue retirado debido a un colapso articular y desaxación en varo con dismetria severa del miembro, a los 26 meses de postoperatorio. Actualmente está siendo tratado mediante osteogénesis por distracción con fijador circular, presentando una buena evolución.

Por su parte, en el caso 10 se debió retirar debido a una necrosis de piel, infección profunda, con exposición del injerto. Luego de varias limpiezas quirúrgicas e intentos de cobertura de las PB, se decidió a los 4 meses de la cirugía inicial retirar el autoinjerto y colocar un espaciador de cemento y fijadores externos (FFEE). En la evolución se reconstruyó con peroné vascularizado, estando actualmente en evolución. En estos 3 casos el tiempo promedio de sobrevida del injerto fue de 21 meses (rango 4-33).

El porcentaje de sobrevida del autoinjerto en nuestra serie fue algo inferior al reportado en la bibliografía. Al igual que en los trabajos publicados por Igarashi et al, Tsuchiya et al ${ }^{13}$ y Paholpak et al $^{32}$, en 2 de 3 retiros de autoinjertos de nuestra serie se trató de injertos OA. Todos los casos que los autores antes mencionados refieren haber retirado el autoinjerto se trató del tipo OA.

Como limitaciones del presente trabajo destacamos el escaso número de pacientes, no obstante dicho número es similar a los trabajos previamente publicados. Además dado lo relativamente nuevo de la técnica y que la comenzamos a desarrollar en el 2015, el seguimiento a largo plazo de estos pacientes aún es bajo, pudiendo subestimar complicaciones a más largo plazo. A pesar de ello nuestro seguimiento medio es similar a dos de los trabajos analizados en la bibliografía (tabla 2). Destacamos que, según nuestro conocimiento, éste trabajo podría corresponder a la primera publicación no occidental con el uso de dicha técnica. 


\section{Conclusiones}

El uso de autoinjerto congelado en NL en reconstrucciones de miembros con patología oncológica músculo-esquelética tras la resección tumoral constituye una muy buena opción para el tratamiento conservador de miembros. Se presenta como una técnica de muy fácil acceso, de bajo costo y mantiene las propiedades de autoconducción y autoinducción. Muestra seguridad en
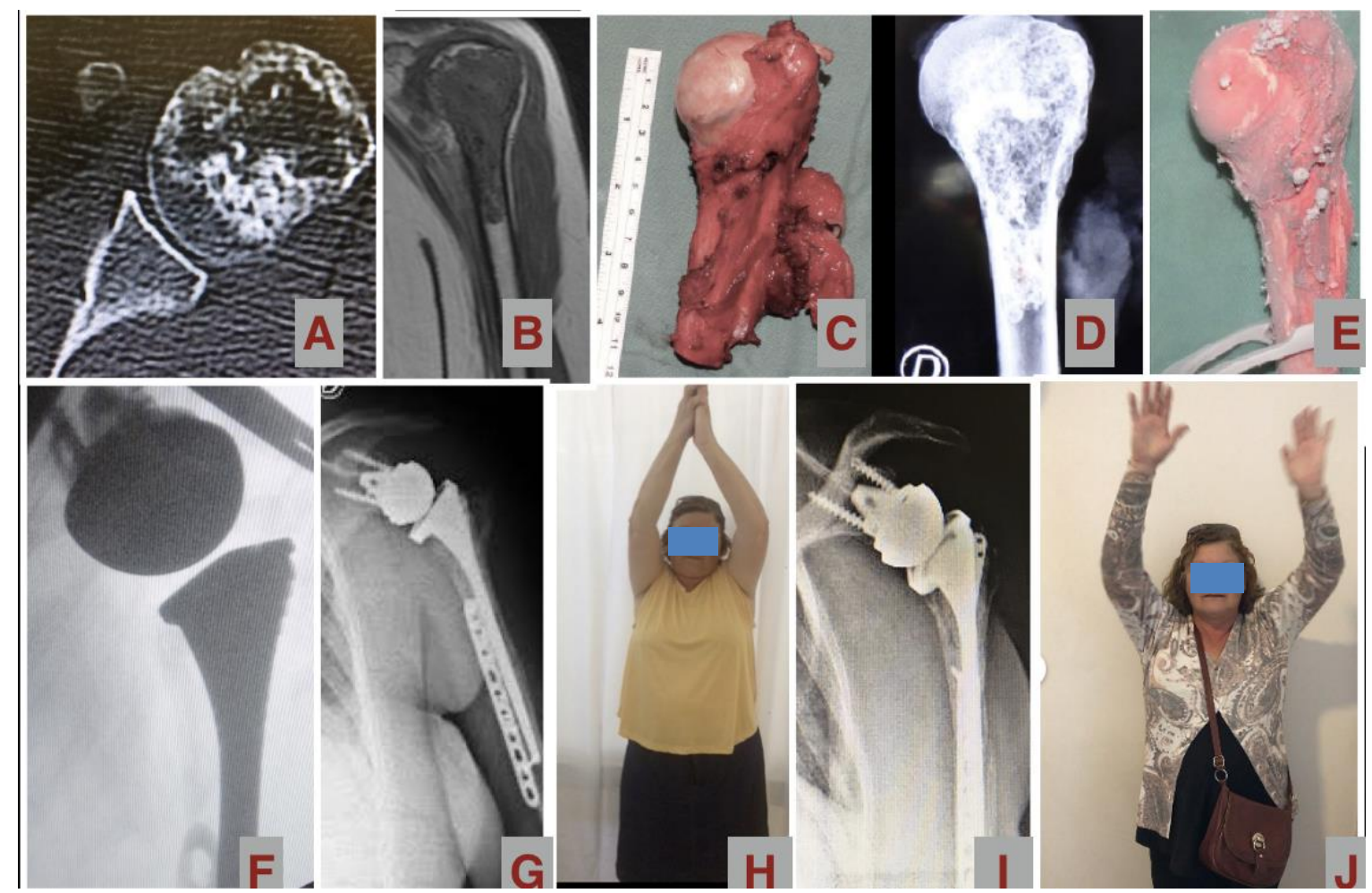

Caso 7. 55 años sexo femenino. Condrosarcoma A) y B) TC y RNM preoperatoria. C) Pieza de resección de húmero proximal. Tipo de Resección OA. D) Radiografía intraoperatoria de la pieza, en la cual se observa que la osteotomia fue realizada con márgenes. E) Autoinjerto Congelado en NL previo a su re-implantación. F) Control intraoperatorio de reconstrucción: "Composite" con Autoinjerto + Artroplastia Reversa de Hombro. G) y H) Radiografía y clínica 4 meses posoperatorio. I) y J) Radiografía y clínica 2 años posoperatorio.

cuanto a la recurrencia local y alto porcentaje de consolidación. Presenta una alta tasa de sobrevida del injerto, siendo mayor en los pacientes en quiénes se realizó injerto intercalar o "composite". El tipo de reconstrucción OA es el que mostró mayor número de complicaciones.

Nuestra cohorte presenta resultados similares a los estudios publicados en cuanto a RL y consolidación, siendo algo menor el porcentaje de sobrevida del injerto. 

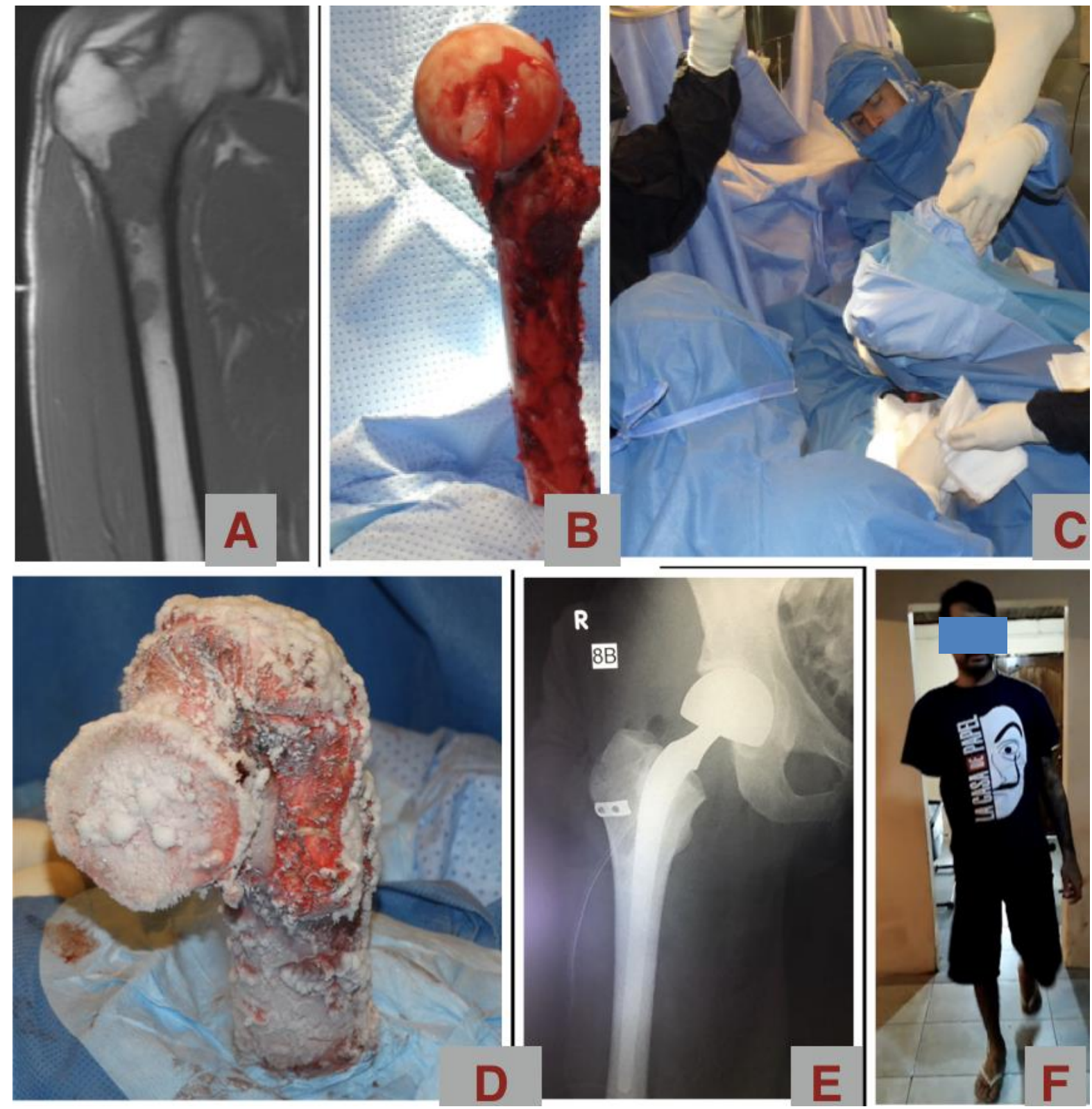

Caso 11. 28 años sexo masculino. Metástasis única en fémur proximal por Sarcoma epiteloide (amputación de brazo derecho 5 años previos). Congelamiento Pediculado "Composite" A) RNM preoperatoria. Extensa lesión en fémur proximal B) Fémur proximal. Aislamiento de la pieza previó a su congelamiento C) Imagen intraoperatoria realizando la rotación del fémur proximal y siendo expuesto al NL. D) Fémur proximal luego de ser congelado en NL. E) Rx posoperatoria. Reconstrucción con prótesis Bipolar. F) 18 meses posoperatorio. Deambulando sin asistencia. 


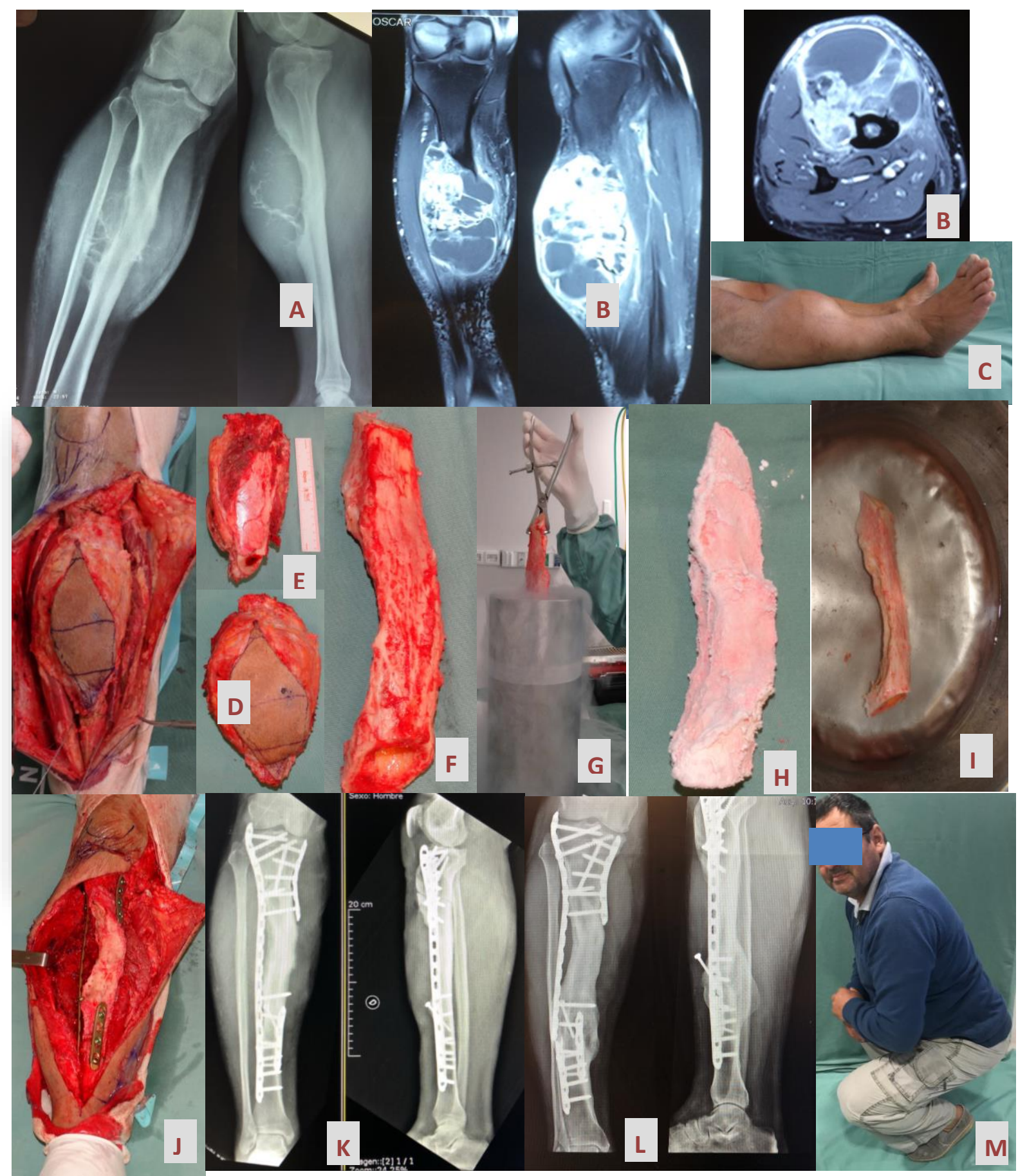

Caso 5. 57 años sexo masculino. Adamantinoma de tibia. A) Radiografías preoperatorias. Extensa lesión que involucra casi la totalidad de la tibia. B) RNM preoperatoria. C) Imagen clínica del miembro del paciente previo al procedimiento. D) Intraoperatorio: cicatriz biópsica emplazada en márgenes de resección previo a la osteotomía distal. E) Pieza de resección con márgenes de partes blandas. F) Pieza de resección luego de ser resecada las partes blandas las cuales se envían a anatomía patológica. Esta pieza está pronta para ser sometida a congelamiento. G) Momento de inmersión de la pieza en NL a - 196 grados Celcius durante 20 minutos. H) Aspecto de la pieza luego del congelamiento, queda 15 minutos a temperatura ambiente siendo calentada progresivamente. I) Continúa el calentamiento progresivo en agua destilada por 10 minutos para luego ser reimplantada. J) Tibia reimplantada y fijación con material de osteosíntesis con tres placas bloqueadas de titanio. L) Radiografías 3 años posoperatorio se objetiva consolidación de ambas líneas osteotomías. M) Imagen clínica del paciente con 3 años posoperatorio. Deambulación sin asistencia y sin dolor. 


\section{Bibliografía}

1- Kotz R, Dominkus M, Salzer-Kuntschik M, Ritschl P, Windhager R, Gadner H, et al. Advances in bone tumor treatment in 30 yeares with respect to survival and limb salvage. A single institution experience. Int Orthop. 2002; 26(4):197-202.

2-Ayerza M, Farfalli G, Aponte-Tinao L, Muscolo D, Muscolo DL. Does increased rate of limb-sparing surgery affect survival in osteosarcoma? Clin Orthop Relat Res. 2010; 468:28549.

3-Nishida J, Shimamura T. Methods of reconstruction for bone defect after tumor excision: a review of alternatives. Medical Science Monitor. 2008; 14:107-13.

4-Capanna R, Scoccianti G, Frenos F, Vilardi A, Beltrami G, Campanacci DA. What was the survival of megaprostheses in lower limb reconstructions after tumor resections? Clin Orthop RelatRes. 2015;473:820-30.

5-Frisoni T, Cevolani L, Giorgini A, Dozza B, Donati D M. Factors affecting outcome of massive intercalary bone allografts in the treatment of tumours of the femur. J Bone Joint Surg. 2012; 94:836-41.

6-Mankin HJ, Gebhardt MC, Jennings LC, Springfield DS, Tomford WW. Long-term results of allograft replacement in the management of bone tumors. Clin Orthop Relat Res. 1996; 324: 86-97.

7-Donati D, Colangeli M, Colangeli S, Di Bella C, Mercuri M. Allograft-prosthetic composite in the proximal tibia after bone tumor resection. Clin Orthop Relat Res. 2008; 466:459-65

8-Yajima, H, Tamai S, Mizumoto S Ono H . Vascularised fibular grafts for reconstruction of the femur. J Bone Joint Surg. 1993; 75: 123-8

9-Watanabe K, Tsuchiya H, Yamamoto N, Shirai T, Nishida H, Hayashi K, et al. Over 10year follow-up of functional outcome in patients with bone tumors reconstructed using distraction osteogenesis. J Orthop Sci. 2013; 18:101-09

10-Manabe J, Ahmed AR, Kawaguchi N, Matsumoto S, Kuroda H. Pasteurized autologous bone graft in surgery for bone and soft tissue sarcoma. Clin Orthop Rel R. 2004; 419: 258-66

11-Uyttendaele D, De Schryver A, Claessens H, Roels H, Berkvens P, Mondelaers W. Limb conservation in primary bone tumors by resection, extracorporeal irradiation and reimplantation. J Bone Joint Surg. 1988; 70: 348-53 
12-Pan KL, Chan WH, Ong GB, , Premsenthil S, Zulkarnaen M, Norlida D. Limb salvage in osteosarcoma using autoclaved tumor-bearing bone. World J Surg Oncol. 2012; 10:105.

13-Tsuchiya H, Wan SL, Sakayama K, Yamamoto N, Nishida H, Tomita K Reconstruction using an autograft containing tumour treated by liquid nitrogen. J Bone Joint Surg Br. 2005; $87: 218-25$.

14-Marcove R, Miller T. The treatment of primary and metastatic localized bone tumors by cryosurgery. Surg Clin North Am. 1969; 49:421-30.

15-Rose P, Morris J. Cryosurgery/cryoablation in musculoskeletal neoplasms: history and state of the art. Musculoskelet Med. 2015; 8:353-60.

16-Yamamoto N, Tsuchiya $\mathrm{H}$, Tomita K. Effects of liquid nitrogen treatment on the proliferation of osteosarcoma and the biomechanical properties of normal bone. J Orthop Sci. $2003 ; 8: 374-80$.

17-Enneking WF, Dunham W, Gebhardt MC, Malawar, Pritchard DJ. A system for the functional evaluation of reconstructive procedures after surgical treatment of tumors of the musculoskeletal system. Clin Orthop Relat Res. 1993; 286: 241-6.

18-Zekry KM, Yamamoto N, Hayashi K, Takeuchi A, Higuchi T, Abe K. Intercalary frozen autograft for reconstruction of malignant bone and soft tissue tumours. Int Orthop. 2017; 41(7):1481-7.

19-Torbert JT, Fox EJ, Hosalkar HS, Ogilvie CM, Lackman RD, et al. . Endoprosthetic reconstructions: results of long-term follow up of 139 patients. Clin Orthop Relat Res. 2005; 438:51-9.

20-Shin DS, Weber KL, Chao EY, An KN, Sim FH. Reoperation for failed prosthetic replacement used for limb salvage. Clin Orthop Relat Res. 1999; 358:53-63.

21-Tomford WW. Transmission of disease through transplantation of musculoskeletal allografts. J Bone Joint Surg Am. 1995; 77(11): 1742-54.

22-Matejovsky Z Jr, Matejovsky Z, Kofranek I. Massive allografts in tumour surgery. Int Orthop. 2006; 30 (6): 478-83.

23-Takata M, Sugimoto N, Yamamoto N, Shirai T, Hayashi K, Nishida H, et al. Activity of bone morphogenetic protein-7 after treatment at various temperatures: freezing vs. pasteurization vs. allograft. Cryobiology. 2011; 63:235-9 
24-Subhadrabandhu S, Takeuchi A, Yamamoto N, Shirai T, Nishida H, Hayashi K, et al Frozen autograft- prosthesis composite reconstruction in malignant bone tumors. Orthopedics. 2015; 38(10):e911-e8.

25-_Tsuchiya H, Nishida H, Srisawat P, Shirai T, Hayashi K, Takeuchi A, et al. Pedicle frozen autograft reconstruction in malignant bone tumors. J Orthop Sci. 2010;15(3): 340-9.

26-_Shimozaki S, Yamamoto N, Shirai T, Nishida H, Hayashi K, Tanzawa Y et al. Pedicle versus free frozen autograft for reconstruction in malignant bone and soft tissue tumors of the lower extremities. J Orthop Sci. 2014; 19(1):156-63.

27-_Miwa S, Takeuchi A, Ikeda H, Shirai T, Yamamoto N, Nishida H, et al. . Prognostic Value of Histological Response to Chemotherapy in Osteosarcoma Patients Receiving Tumor-Bearing Frozen Autograft. 2013; PLoS ONE 8(8): e71362.

28-Nishida H, Tsuchiya H, Tomita K. Reimplantation of tumour tissue treated by cryotreatment with liquid nitrogen induces anti-tumour activity against murine osteosarcoma. J Bone Joint Surg Br. 2008; 90:1249-1255.

29-Nishida H, Yamamoto N, Tanzawa Y, Tsuchiya H. Cryoimmunology for malignant bone and soft-tissue tumors. Int J Clin Oncol. 2011; 16:109-17.

30-Nishida H, Yamamoto N, Shirai T, Tsuchiya H. Cryotreatment against metastatic renal cell bone tumour reduced multiple lung metastases. Anticancer Research. 2011; 31: 2927-30.

31-_Tanzawa Y, Tsuchiya H, Shirai T, Hayashi K, Yo Z, Tomita K. Histological examination of frozen autograft treated by liquid nitrogen removed 6 years after implantation. J Orthop Sci. 2008. 13: 25964.

32-_ Paholpak P, Sirichativapee W, Wisanuyotin T, Kosuwon W, Jeeravipoolvarn P. Clinical results of primary malignant musculoskeletal tumor treated by wide resection and recycling autograft reconstruction using liquid nitrogen. Asia Pac J Clin Oncol. 2015; 11:114-20.

33-_Igarashi K, Yamamoto N, Shirai T, Hayashi K, Nishida H, Kimura H, et al. The long-term outcome following the use of frozen autograft treated with liquid nitrogen in the management of bone and soft tissue sarcomas. Bone Joint J. 2014; 96:555-61.

34-Rahman MA, Bassiony A, Shalaby H. Reimplantation of the resected tumour-bearing segment after recycling using liquid nitrogen for osteosarcoma. Int Orthop. 2009; 33(5): 136570 . 